\title{
Identifying and Managing Pain in People with Alzheimer's Disease and Other Types of Dementia: A Systematic Review
}

\author{
Bettina S. Husebo ${ }^{1,2} \cdot$ Wilco Achterberg ${ }^{1,3} \cdot$ Elisabeth Flo $^{1}$
}

Published online: 30 May 2016

(c) The Author(s) 2016. This article is published with open access at Springerlink.com

\begin{abstract}
Background and Objective Pain in patients with Alzheimer's disease is a complex issue; these patients suffer from the common causes of acute and chronic pain, and some also have neuropathic or nociceptive pain. Whatever the mechanism of pain in these patients, their pain will require careful assessment and management, to insure the correct type and level of analgesia is given. The objective of this systematic review was the identification of studies that have investigated the efficacy of different analgesics on pain intensity or pain-related behavior during nursing home stay and at the end of life.

Methods A search using pain, pain treatment, and dementia MESH terms and keywords was conducted (October 15, 2015) in MEDLINE, EMBASE, PsychINFO, CINAHL, and Cochrane libraries.

Results Our search yielded 3138 unique hits, published between 1990 and October 2015. We read titles and
\end{abstract}

Electronic supplementary material The online version of this article (doi:10.1007/s40263-016-0342-7) contains supplementary material, which is available to authorized users.

Bettina S. Husebo

bettina.husebo@igs.uib.no

1 Department of Global Public Health and Primary Care, Centre for Elderly and Nursing Home Medicine, University of Bergen, Bergen, Norway

2 Section for Nursing Home Medicine, Municipality of Bergen, Bergen, Norway

3 Department of Public Health and Primary Care, Leiden University Medical Center, Leiden, The Netherlands abstracts, identified 124 papers for full-text evaluation, and included 12 papers to reflect and synthesize the following questions: (1) Which pain assessment tools for people with dementia are responsive to change in pain intensity scores? (2) Which analgesics are efficacy-tested by controlled trials including people with dementia living in nursing homes, including at the end of life? (3) Which outcome measures have been used to identify pain, pain behavior, and/or treatment efficacy in people with dementia?

Conclusion Despite increased use of analgesics, pain is still prevalent in people with dementia. Validated pain tools are available but not implemented and not fully tested on responsiveness to treatment. Official guidelines for pain assessment and treatment addressing people with dementia living in a nursing home are lacking. The efficacy of analgesic drug use on pain or neuropsychiatric behavior related to dementia has been hardly investigated.

\section{Key Points}

This systematic review adds to previous reviews of the assessment and treatment of pain in people with dementia, with a particular focus on studies investigating the direct efficacy of analgesics on pain intensity.

The review underlines current challenges: thorough pain assessment is poorly implemented,

observational pain instruments are rarely tested for responsiveness; therefore, sound evaluation of pain management strategies is lacking and evidence for efficacy and safety of analgesics is largely missing. 


\section{Introduction}

It is widely acknowledged that the assessment and treatment of acute and persistent pain is a complex entity in nursing home patients with Alzheimer's disease [1]. There are two extreme endpoints related to these challenges. On one side, the prevalence of undiagnosed and untreated pain is described as unacceptably high in the primary health care system, with reported prevalence up to $32 \%$ in homedwelling people with dementia [2] and about $60 \%$ in nursing home patients with and without dementia [3, 4]. On the other hand, recent reports document increasing analgesic drug prescription trends, especially in Scandinavia [5-7], regardless of the potential side effects [8] and the prevalent polypharmacy in these patients. This picture is getting even more complex in light of the individual persons who experience the pain. Most of these people develop changes in their physical function caused by multimorbidity and increased vulnerability [9]. They have high needs for motivated and competent nursing home staff [10], implementation of research-based knowledge [11], routine use of validated pain assessment tools [12, 13], and guidelines for appropriate analgesic treatment [14]. This complex clinical situation is illustrated in the case of Mr. K (Fig. 1a, b).

Alzheimer's dementia (AD) and vascular dementia $(\mathrm{VaD})$ are age-related diseases that have a major impact on health care resources. At present, 35 million people worldwide have dementia, with an expected increase to 115 million by 2050 [15]. In advanced stages of dementia, AD is often combined with $\mathrm{VaD}$ to mixed dementia (AD-VaD), suggested to be the most prevalent in nursing home patients $[4,16]$. These people are more prone to central, neuropathic pain, because lesions in the white matter substance disturb the connections in the pain system [17].

Despite over a century of scientific endeavor, effective curative treatment options for these diseases are still lacking, and increasing frailty and disabilities require institutional care for more and more individuals. During the course of the disease, the slight progress of AD substantially affects the motivational and cognitive structures of the pain network, usually responsible for properly assigning the meaning to pain $[1,12,13]$.

In addition to memory disturbances, dementia is commonly accompanied by neuropsychiatric symptoms [18] such as agitation and aggression, depression and apathy, eating and appetite disturbances, and sleeping disorders [19]. It is suggested that the causes of these symptoms are multifactorial, based on chemical, anatomical, and transmitter changes in the brain and/or related to unmet needs. One important trigger for neuropsychiatric symptoms may be undiagnosed and untreated pain [20]. However, proper assessment and treatment of pain in people with dementia is hampered by the challenge that those in advanced stages of the disease are no longer able to describe their suffering and to give precise self-reports regarding the intensity, location, and duration of the pain [1]. In addition, they are not able to report appropriate pain relieving effects or side effects in connection with the pain treatment [13].

General agreement exists that proper assessment of pain is a prerequisite for proper treatment. During this process, if self-report is no longer possible, a validated, reliable, and responsive observational, behavioral pain assessment tool is required. This means that a proxy rater, usually a nurse who knows the patient over time, should be able to interpret the patient's behavior and transfer it into a measure of pain presence and preferably also into pain intensity [16, 21]. During the last 25 years, about 30 tools have been developed and tested to assess pain in people with dementia. Although several review articles present overviews over such tools [22-29], there is no general agreement on which instrument should be recommended. We suggest, however, that clinicians and researchers worldwide appreciate different tools, possibly in relation to the country in which the respective instrument has been developed, the availability in that specific country and language, and the recommendations by national and regional procedures and guidelines.

Dementia is a life-limiting disease that requires treatment and care in nursing homes or hospitals for almost all patients at the end of life [30]. This means that the identification and management of pain in the final weeks and hours should also be addressed as a key topic [31].

Nonetheless, the effectiveness of a pain treatment strategy during nursing home stay and at the end of life cannot be guaranteed by the amount of analgesic drug prescriptions alone. The way to succeed includes proper assessment, treatment, and evaluation of the treatment effect by re-assessment, again using a validated pain tool. Clearly, this requires nursing home staff who are skilled in conducting these procedures and a physician who is a specialist on dementia assessment and communicates well with the multidisciplinary team.

Although there have been other systematic reviews on pain in dementia, for instance, on the reliability and validity of pain scales, this one is the first to look at important pain management features, such as outcome measures for pain treatment, responsiveness of pain observation scales, and efficacy of pain medication. The main goal of this systematic review is the identification of studies that directly investigate the efficacy of different analgesics on pain intensity or typical behavior that might be related to pain in people with dementia, during nursing home stay and at the end of life. In order to evaluate the quality of the included studies, we additionally did an 
Fig. 1 a The case of Mr. K. b Conclusion of the case of $\mathrm{Mr}$. K. MOBID-2 MobilizationObservation-BehaviorIntensity-Dementia-2 Pain Scale a Mr. K. is 78 years old. For the last 20 months, he has been living in a Norwegian nursing home after he developed Alzheimer's disease, about eight years ago. Mr. K. lost his speech ability due to a stroke; this incident also caused a paresis in his right arm. With support by staff, he is able to leave his bed and sit in his chair. Recently, the responsible physician came to Mr. K.'s nursing home ward. When she entered the unit, she heard a patient shouting loudly out of the room. She asked the staff what was going wrong. A nurse answered: "Well, Mr. K. is screaming a lot; the screaming started about 14 days ago. We discussed his psychiatric problems in our team; we think that he pretends to be in pain. Mr. $\mathrm{K}$. is very aggressive and uncooperative during care. Today, he already received a sedative drug, but it hasn't worked." When the nurse together with the physician entered the room of Mr. K., he was restlessly sitting in a wheelchair. After transfer into his bed, a systematic examination (using the procedure of the MOBID-2 Pain Scale, yielding a score of 8) of his body revealed that Mr. K. had a small, red, and infected sacral pressure ulcer.

In addition, gentle guided movements of his limbs revealed painful stiffness in his right shoulder and tenderness in the muscles of the right arm, with upcoming contracture. During the consultation, Mr. K. reacted with more restlessness, resistance, and vocalization. It was obvious that he was no longer able to describe his pain but responded by changing his usual behavior.

b Taking all the observations into account, the physician organized a multidisciplinary meeting to discuss Mr. K.'s behavior. The primary contact, physiotherapist, and music therapist were present. They all discussed their perspectives. A systematic work plan was developed including the wheelchair function with a comfort pillow, physical therapy with gentle massage of the shoulders and greater patience during care. Twice a week, Mr. K. was invited to participate in a group of music therapy.

His drug pain regime was changed from paracetamol $1 \mathrm{~g}$ in the evening to paracetamol $1 \mathrm{~g}$ every eight hours. The neuropathic pain suggested in his right shoulder and arm was treated with pregabalin (Lyrica $\left.{ }^{\circledR}\right) 25 \mathrm{mg}$ in the evening, with increased dosage of $25 \mathrm{mg}$ x 2, after 7 days. However, Mr. K. reacted with confusion and edema in both of his legs; thus pregabalin was reduced. Instead, the physician started with buprenorphine transdermal-system (Norspan $\left.{ }^{\circledR}\right) 5 \mu \mathrm{g} /$ hour/changed every $7^{\text {th }}$ day. Through-out this period Mr. K. was examined at least once a week. During the next few days and weeks, the patient was more relaxed, less aggressive, and the wound was healing. Again using the MOBID-2 Pain Scale, pain intensity scores decreased to 3 and it was possible to withdraw buprenorphine. Pregabalin was continued in the lowest dosage administered in the evening. Close monitoring and regular control of the pain condition was still required. However, the daily situation was inconsistent for Mr. K.; not everything was always optimal, but things were much better. The relatives also felt it easier to come to visit. Mr. K. showed more pleasure when he met his little grandchild. updated search based on recent systematic reviews of behavioral pain assessment tools in people with dementia published by the authors $[1,13,21,32]$. The purpose of this was to be able to ascertain whether the observational pain tools used in the included efficacy trials were tested for responsiveness. Thus, we will explore the following questions:

- Which analgesics are efficacy-tested by clinical or randomized controlled trials in people with dementia?

- Which outcome measures have been used to identify pain, pain behavior, and/or pain treatment efficacy?
- Which of the pain assessment tools used for people with dementia are responsive to change in pain intensity scores, and thus, adequate to use in a pain treatment efficacy trial?

\section{Method}

This systematic review of the literature aims to gather fully randomized controlled studies with comparator groups or open studies with more than ten participants that 
investigate the efficacy of treating pain on pain intensity scores and/or behavioral disturbances and neuropsychiatric symptoms in people with dementia. By this, we will describe analgesic interventions and assessment procedures related to the intervention. We recognize that non-pharmacological interventions in many instances are the first choice treatment of behavioral disturbances. However, this is beyond the scope of this current review.

\subsection{Literature Search}

Assisted by the University Library in Bergen, PICO-based [problem/population (P), intervention (I), comparison (C) and outcomes $(\mathrm{O})$ (Table 1)] literature searches were conducted in October 2015, covering medical peer-reviewed publications (original papers and systematic reviews) published between 1990 and October 2015. We searched MEDLINE, EMBASE, CINAHL, PsychINFO and Cochrane libraries (Fig. 2). Key words included MESH terms and phrases synonymous with "pain," "treatment," and "dementia" (the online appendix—see the electronic supplementary material-provides a complete overview of the different MESH terms). The literature searches were then collated and all the authors discussed inclusion of publications (see Table 1 for a description of inclusion and exclusion criteria). To guarantee that we recognized all the relevant publications, two different researchers (B. S. H., E. F.) independently selected the relevant studies. Only articles in the English language were included. On the basis of the exclusion criteria listed in Table 1, the authors screened potential manuscripts at the abstract level and applied the review protocol of described inclusion and exclusion criteria on selected full texts; we also searched through the reference lists of the full-text publications. The authors discussed in group meetings all manuscripts read in full text and borderline exclusion cases.

In order to extract and synthesize the content of the manuscripts included in this review, the authors agreed on the content to be extracted and developed a data-extraction table with cells for content to be extracted from the manuscripts. The table was piloted and discussed in the group. A minimum of two of the co-authors read through each publication independently and then verified the data extraction in a discussion. Any unclear material was raised in group discussions. When applicable, the following was extracted from each publication: full manuscript reference, number of participants, study design and method, type of intervention (specifically, type of pain medication) and control condition, time to follow-up, and study setting and outcomes. Finally, the authors agreed upon the organizing themes of Tables 2, 3, and 4, and the grading of evidence according to an adapted Oxford Centre for Evidence-based Medicine-Levels of Evidence document. This system allows author to grade both studies and reviews on the basis of quality factor such as sample homogeneity, followup, definition of comparison group, and use of validated and relevant tests. The ten different levels of evidence defined in the system are then translated into grades of recommendation (A-D).

We had a special focus on articles that described the responsiveness testing of observational pain assessment instruments. With responsiveness, we are not referring to standard error of mean, but to studies demonstrating that the instrument is responsive to pain treatment. However, general publications that focused on the development, validation, and testing of pain assessment tools were excluded, as these have previously been described in detail $[1,13,21,32]$.

\section{Results}

After exclusion of the doublets, the systematic search generated 3135 unique hits. Subsequently, B.S.H. and E.F. read the titles and abstracts and identified 124 papers for full-text evaluation, of which 79 manuscripts were potential primary studies and 49 were potential systematic

Table 1 Defined PICO-based search strategy, and listed exclusion criteria

\begin{tabular}{|c|c|}
\hline Population & Patients with Alzheimer's disease or undefined dementia \\
\hline Intervention & Pain treatment defined as a pharmacological intervention in which the medications employed are named and dosages listed \\
\hline Comparison & All studies using standard care as usual group comparison or other valid comparison alternatives before/after comparison \\
\hline Outcome & $\begin{array}{l}\text { Only studies employing quantitative measures and analyses were included. Outcomes included assessment of pain (yes/no or } \\
\text { pain intensity) as well as assessment of pain-related behavior, e.g., neuropsychiatric symptoms }\end{array}$ \\
\hline $\begin{array}{l}\text { Exclusion } \\
\text { criteria }\end{array}$ & $\begin{array}{l}\text { (1) Qualitative studies, (2) single case studies and those including less than ten patients, (3) methodology papers, (4) study } \\
\text { protocols, (5) animal studies, (6) non-pharmacological studies, (7) deep brain stimulation and other experimental studies, and } \\
\text { (8) conference abstracts. On the basis of these exclusion criteria, the authors screened potential manuscripts at the abstract } \\
\text { level and applied the review protocol of described inclusion and exclusion criteria on selected full texts; we also searched } \\
\text { through the reference lists of the full-text publications }\end{array}$ \\
\hline
\end{tabular}

PICO problem/population, intervention, comparison and outcomes 
Fig. 2 PRISMA based flow chart over the literature search, selection and review process

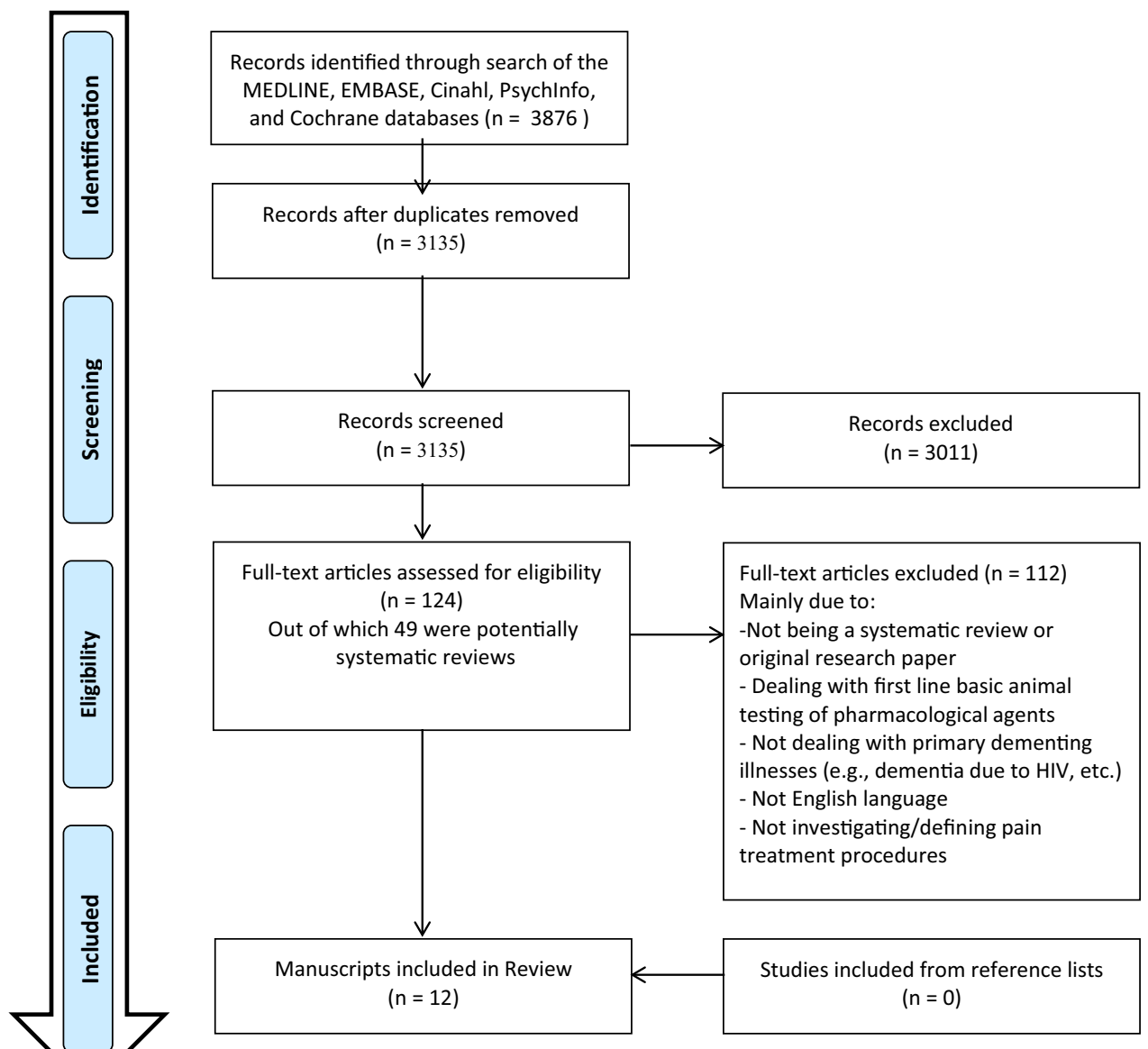

reviews. A search through the reference lists of the included papers yielded no further publications. Eight studies yielding 12 publications met the selection criteria (Fig. 2). These were published between 2003 and 2014, with patient sample size ranging from 24 to 352 , and number of nursing homes ranging from one to 18 . Three studies were published by researchers from the USA, two from the Netherlands, and one study each from Finland, Italy, and Norway. The quality grading of evidence (grade A-D) is indicated in Tables 2 and 3. Five of these studies included typical nursing home patients [33-37], two trials included dying people [38, 39], and one study had an experimental design with home-dwelling participants [40]. In four studies [33, 36, 37, 39], the type of dementia was not specified among included participants, whereas one study included only people with AD [40]; in another, almost half (43\%) of the patients had $\mathrm{AD}$ [38]. The remaining two studies involved people with diagnoses of $\mathrm{AD}$ or $\mathrm{VaD}$ [34] and multi-infarct, or degenerative dementia [35]. Six studies investigated the efficacy of a monotherapy with paracetamol (acetaminophen), morphine, vitamin $\mathrm{D}$, and lidocaine gel, while another two studies used several treatment options and a stepwise protocol of treating pain (SPTP) (Tables 2, 3).

We did not find intervention studies that included treatment with oral or topical nonsteroidal anti-inflammatory drugs (NSAIDs), anticonvulsants, tricyclic antidepressants, tramadol, codeine, or buprenorphine as monotherapy.

Further, we found four papers that described the responsiveness testing of observational pain assessment tools (Table 4).

\subsection{Which Pain Assessment Tools for People with Dementia are Responsive to Change in Pain Intensity Scores?}

Responsiveness is the "ability of an instrument to detect change over time in the construct to be measured" [41]. Thus, a responsive observational pain tool is a prerequisite for proper treatment of pain in people with dementia to address the change in the total pain intensity score after pain treatment has been initiated [21]. Psychometric property testing of such a tool should follow recent COnsensus-based Standards for the selection of health 
Table 2 Clinical studies investigating the efficacy of analgesic treatment on pain intensity or typical behavior of pain in people with advance dementia

\begin{tabular}{|c|c|c|c|c|c|}
\hline Pain medication & $\begin{array}{l}\text { Authors, } \\
\text { year, } \\
\text { nationality, } \\
\text { grade }^{\mathrm{a}}\end{array}$ & Population & Method & Outcomes & Findings \\
\hline \multirow[t]{2}{*}{$\begin{array}{l}\text { Paracetamol/ } \\
\text { acetaminophen }\end{array}$} & $\begin{array}{l}\text { Chibnall } \\
\text { et al. [35], } \\
\text { 2005, } \\
\text { USA, B }\end{array}$ & $\begin{array}{l}25 \mathrm{NH} \text { patients with } \\
\text { moderate-to-severe } \\
\text { dementia (degenerative } \\
\text { 13, AD 4, multi-infarct } \\
\text { dementia 4) }\end{array}$ & $\begin{array}{l}\text { Randomized, double-blind, } \\
\text { placebo-controlled, crossover } \\
\text { trial; } 4 \text { weeks placebo, } 4 \text { weeks } \\
\text { treatment }\end{array}$ & $\begin{array}{l}\text { DCM } \\
\text { CMAI; } \\
\text { no pain } \\
\text { tool }\end{array}$ & $\begin{array}{l}\text { Patients were more active and spent } \\
\text { more time in social surroundings } \\
\text { and interacting with others } \\
\left(p<0.05, \eta^{2} \text { from } 0.15 \text { to } 0.25\right) \text {; no } \\
\text { effect on agitation, emotional well- } \\
\text { being or as-needed psychotropic } \\
\text { medication }\end{array}$ \\
\hline & $\begin{array}{l}\text { Buffum } \\
\text { et al. [34], } \\
\text { 2004, } \\
\text { USA, B }\end{array}$ & $\begin{array}{l}39 \mathrm{NH} \text { patients with } \\
\text { dementia }(\mathrm{AD} / \mathrm{VaD}) \text { and } \\
\text { pain rel. degenerative } \\
\text { joint disease; mean } \\
\text { MMSE } 4.3 \text { and GDS } 5.7\end{array}$ & $\begin{array}{l}\text { Double-blind, placebo-controlled, } \\
\text { crossover } 4 \text {-week trial } \\
\text { investigating scheduled } \\
(650 \mathrm{mg} \times 4 / \text { day }) \text { vs. as-needed } \\
\text { acetaminophen administration }\end{array}$ & DS-DAT & $\begin{array}{l}\text { No differences were found between } \\
\text { scheduled or as-needed } \\
\text { acetaminophen application; } \\
2600 \mathrm{mg} / \text { day acetaminophen is } \\
\text { inadequate for NH patients with } \\
\text { degenerative joint disease }\end{array}$ \\
\hline $\begin{array}{l}1 \% \text { solution of } \\
\text { lidocaine, a } \\
\text { local } \\
\text { anesthetic }\end{array}$ & $\begin{array}{l}\text { Benedetti } \\
\text { et al. [40], } \\
\text { 2006, I, B }\end{array}$ & $\begin{array}{l}28 \text { people with AD; mean } \\
74 \text { years; baseline MMSE } \\
22-25 \text {, after } 1 \text { year } 13-20\end{array}$ & $\begin{array}{l}\text { Placebo-related analgesic study } \\
\text { with open (expected)/hidden } \\
\text { (unexpected) paradigm lidocaine } \\
\text { gel }\end{array}$ & $\begin{array}{l}\text { NRS-self } \\
\text { rating }\end{array}$ & $\begin{array}{l}\text { People with dementia/reduced FAB } \\
\text { show reduced placebo effect of } \\
\text { analgesic treatment [post hoc } \\
\text { Tukey test, } q(42)=13.524, \\
p<0.001 \text { ] . Analgesics should be } \\
\text { increased to compensate for the } \\
\text { loss of placebo effect }\end{array}$ \\
\hline \multirow[t]{2}{*}{ Morphine } & $\begin{array}{l}\text { Manfredi } \\
\text { et al. [36], } \\
\text { 2003, } \\
\text { USA, B }\end{array}$ & $\begin{array}{l}47 \text { people with dementia } \\
\text { (unknown type) from one } \\
\text { NH (1999-2001); mean } \\
86 \text { years; MMSE }<20 ; \\
\text { CMAI } \geq 40\end{array}$ & $\begin{array}{l}\text { All patients received placebo for } \\
4 \text { weeks followed by treatment } \\
\text { with oxycodone } 20 \mathrm{mg} / \text { day or } \\
\text { morphine } 20 \mathrm{mg} / \text { day }\end{array}$ & $\begin{array}{l}\text { CMAI; } \\
\text { no pain } \\
\text { tool }\end{array}$ & $\begin{array}{l}25 \text { patients completed the study; no } \\
\text { differences in agitation between } \\
\text { placebo and opioid phases, though } \\
\text { patients } \geq 85 \text { years old }(N=13) \\
\text { were less agitated (CMAI score } \\
-6.4 ; 95 \% \text { confidence interval } \\
-10.96 \text { to }-1.8) \text {. No group } \\
\text { differences in sedation; high drop- } \\
\text { out rate }\end{array}$ \\
\hline & $\begin{array}{l}\text { Klapwijk } \\
\text { et al. [39], } \\
\text { 2014, NL, } \\
\text { C }\end{array}$ & $\begin{array}{l}24 \text { dying patients with most } \\
\text { severe dementia (type } \\
\text { unknown); two NHs; } \\
\text { median age } 91 \text { years }\end{array}$ & $\begin{array}{l}\text { Observational study of the last days } \\
\text { of life; small sample size; pain } \\
\text { instruments not validated for EoL } \\
\text { care }\end{array}$ & $\begin{array}{l}\text { PAINAD } \\
\text { DS-DAT } \\
\text { EOLD- } \\
\text { CAD } \\
\text { MMSE }\end{array}$ & $\begin{array}{l}\text { Mean } 4.3 \text { observations per patient; all } \\
\text { participants received morphine } \\
\text { (dosage not available). Low } \\
\text { symptom burden but direct effect } \\
\text { of morphine was not estimated }\end{array}$ \\
\hline Vitamin D & $\begin{array}{l}\text { Björkman } \\
\text { et al. [33], } \\
\text { 2008, } \\
\text { Finland, } \\
\text { B }\end{array}$ & $\begin{array}{l}202 \text { NH patients with pain } \\
\text { and dementia (type } \\
\text { unknown); mean } \\
85 \pm 7 \text { years; CPS } \\
4.9 \pm 1.4 \text {, range } 1-6\end{array}$ & $\begin{array}{l}\text { Randomized, double-blind placebo- } \\
\text { controlled 6-month trial with } \\
\text { vitamin D supplement in three } \\
\text { groups: 0, } 400 \text { or } 1200 \mathrm{IU} \\
\text { cholecalciferol }\end{array}$ & $\begin{array}{l}\text { RAI } \\
\text { PAINAD } \\
\text { DS-DAT } \\
\text { CPS }\end{array}$ & $\begin{array}{l}38-84 \% \text { of the patients were in pain; } \\
\text { vitamin D deficiency was not } \\
\text { associated to pain or pain behavior; } \\
\text { prevalence of painlessness or pain } \\
\text { scores not changed after vitamin D } \\
\text { treatment }\end{array}$ \\
\hline
\end{tabular}

$A D$ Alzheimer's disease, CMAI Cohen-Mansfield Agitation Inventory [86], CPS Cognitive Performance Scale, DCM Dementia Care Mapping, DS-DAT Discomfort Scale-Dementia of Alzheimer Type [87], EoL end-of-life, EOLD-CAD End-of-Life in Dementia-Comfort Assessment in Dying [88], FAB Frontal Assessment Battery [89], GDS Global Deterioration Scale [90], I Italy, MMSE Mini-Mental State Examination [95], $N H$ nursing home, $N L$ Netherlands, NRS Numerical Rating Scale, PAINAD Pain Assessment in Advanced Dementia [91], RAI Resident Assessment Instrument, rel related to, $\mathrm{VaD}$ vascular dementia

${ }^{\text {a }}$ Quality grade according to the Oxford grading of evidence

Measurement INstruments (COSMIN) recommendations [41]. Four validation studies with comparator groups including between 91 and 352 patients have evaluated the responsiveness of several observational pain assessment tools [42-45] (Table 4).

Morello et al. explored the psychometric properties of the Elderly Pain Caring Assessment 2 (EPCA-2), which rates the pain intensity in non-communicating elderly people by eight behavioral items with two dimensions: the signs outside and during care giving [43]. Patients $(N=340)$ from three French hospitals were assessed by experienced observers (pairs of doctors and nurses) for 7 days, in order to study the patients' behavior in the absence of pain and during a presumed state of pain (before and after taking analgesics). The responsiveness of the EPCA-2 was demonstrated by changes of pain intensity scores that correlated highly to other change scores of the Global Clinical Score (GCS) and analgesic drug use (opioid 
Table 3 Clinical studies investigating the efficacy of analgesic combination therapy in people with advance dementia

\begin{tabular}{|c|c|c|c|c|c|}
\hline Pain medication & $\begin{array}{l}\text { Authors, year, } \\
\text { references, } \\
\text { nationality, } \\
\text { grade }^{\mathrm{a}}\end{array}$ & Population & Method & Outcomes & Findings \\
\hline $\begin{array}{l}\text { Paracetamol/ } \\
\text { acetaminophen, } \\
\text { NSAIDs, oral or } \\
\text { parenteral } \\
\text { opioid }\end{array}$ & $\begin{array}{l}\text { Hendriks } \\
\text { et al. } \\
2014-2015 \text {, } \\
{[38,48] \text {, }} \\
\text { NL, C }\end{array}$ & $\begin{array}{l}330 \text { dying people with } \\
\text { dementia from } 34 \mathrm{NHs} \\
(2007-2011) ; 43 \% \\
\text { had AD }\end{array}$ & $\begin{array}{l}2 \text { weeks after death } \\
\text { retrospective } \\
\text { questionnaire to } \\
\text { investigate } \\
\text { association between } \\
\text { QoL and pain }\end{array}$ & $\begin{array}{l}\text { 11-item } \\
\text { QUALID; } \\
\text { no pain } \\
\text { tool }\end{array}$ & $\begin{array}{l}\text { In the last week of life, } 52 \% \text { were } \\
\text { found to be in pain; no differences } \\
\text { between patients between advanced } \\
\text { and less advanced dementia; } 77 \% \\
\text { received opioids ( } 90 \mathrm{mg} / 24 \mathrm{~h} ; 88 \% \\
\text { by injection); } 43 \% \text { opioid } \\
\text { monotherapy, } 57 \% \\
\text { opioids }+ \text { paracetamol. Cannot } \\
\text { determine the most effective } \\
\text { treatment of symptoms }\end{array}$ \\
\hline $\begin{array}{l}\text { Paracetamol, } \\
\text { morphine, } \\
\text { buprenorphine, } \\
\text { and/or } \\
\text { pregabalin }\end{array}$ & $\begin{array}{l}\text { Husebo et al. } \\
\text { 2011-2014, } \\
\text { [7, 37, 49, } \\
50], \text { N, A }\end{array}$ & $\begin{array}{l}352 \mathrm{NH} \text { patients with } \\
\text { advanced dementia } \\
\text { (type unknown) and } \\
\text { agitation from } 60 \mathrm{NH} \\
\text { clusters }\end{array}$ & $\begin{array}{l}\text { CRCT over } 12 \text { weeks; } \\
\text { NH clusters were } \\
\text { randomized to an } \\
\text { SPTP }\end{array}$ & $\begin{array}{l}\text { CMAI } \\
\text { NPI-NH } \\
\text { MMSE } \\
\text { MOBID-2 } \\
\text { Pain Scale } \\
\text { ADL }\end{array}$ & $\begin{array}{l}\text { Agitation reduced in the SPTP group } \\
\text { (average reduction } 17 \% \\
(p<0.001,95 \% \text { confidence } \\
\text { interval }-3.7 \text { to }-10.3)[37] . \\
\text { Verbally agitated behaviors, } \\
\text { physically non-aggressive } \\
\text { behaviors, and aggressive behaviors } \\
\text { improved after } 8 \text { weeks }(p<0.001, \\
p=0.008, p=0.037, \text { respectively) } \\
{[50] . \text { Mood syndrome improved }} \\
(p<0.001) \text {, including depression } \\
(p=0.025), \text { apathy }(p=0.017), \\
\text { sleep }(p=0.050) \text {, and appetite } \\
(p=0.005) \text { [49]. Pain reduced in } \\
\text { SPTP group compared with control } \\
(p<0.001) \text { at week } 8, \text { and pain } \\
\text { scores worsened during washout } \\
(p=0.022) \text {. Acetaminophen } \\
\text { improved ADL function }(p=0.02) \\
\text { [7] }\end{array}$ \\
\hline
\end{tabular}

$A D$ Alzheimer's disease, $A D L$ Activities of Daily Living [52], CMAI Cohen-Mansfield Agitation Inventory [86], $C R C T$ cluster randomized clinical trial, MMSE Mini-Mental State Examination [95], MOBID Mobilization-Observation-Behavior-Intensity-Dementia [42], $N$ Norway, $N H$ nursing home, $N L$ Netherlands, NPI-NH Neuropsychiatric Inventory-Nursing Home version [92], NSAID nonsteroidal anti-inflammatory drug, QoL quality of life, QUALID Quality of Life in Late-Stage Dementia [93], SPTP stepwise protocol of treating pain

${ }^{a}$ Quality grade according to the Oxford grading of evidence

subgroup $N=112$, non-opioid subgroup $N=171$ ), compared with the non-analgesic subgroup $(N=57)$.

Cohen-Mansfield conducted an open pain treatment trial to investigate the responsiveness of nine observational pain tools after pain treatment in 121 patients with dementia living in four nursing homes in Maryland [44]. Patients $(N=36)$ with pain at baseline were treated following an analgesic protocol of paracetamol, oxycodone, or oxycontin. The study had three comparator groups: patients who were in pain at baseline and treated with the analgesic protocol; those with pain at baseline, but the primary caregiver did not follow the protocol; and those without pain $(N=58)$. Patients who followed the analgesic treatment protocol had a significant reduction of pain compared with the other two groups. The Pain Assessment in
Noncommunicative Elderly persons (PAINE) and Pain Assessment for the Dementing Elderly (PADE) pain tools were highlighted as the most responsive to assess the change in pain intensity scores.

The third study, by Rat et al. conducted an open pain treatment trial to investigate the responsiveness of the acute pain instrument Algoplus ${ }^{\circledR}$ in 91 French hospital patients with dementia [45]. Acute pain was defined by a list of pain-inducing situations, such as traumatic injuries, fractures, abdominal pain, or bedsores. Algoplus ${ }^{\circledR}$ sensitivity to change was tested during movement and after starting acute pain management with World Health Organization (WHO) level I drug, i.e., non-opioids (37 \%), II drug, i.e., mild opioids (30\%), III drug, i.e., strong opioids $(23 \%)$, and others $(3 \%)$. Paired comparisons by Wilcoxon's 
Table 4 Responsiveness of pain assessment instruments to assess change in pain intensity after initiated pain treatment in people with dementia

\begin{tabular}{|c|c|c|c|c|}
\hline $\begin{array}{l}\text { Author, } \\
\text { year, } \\
\text { reference }\end{array}$ & Pain tool & $\begin{array}{l}\text { Setting and } \\
\text { participants }\end{array}$ & Method & Findings \\
\hline $\begin{array}{l}\text { Morello } \\
\text { et al. } \\
2007,[43]\end{array}$ & EPCA-2 & $\begin{array}{l}340 \text { NVC-OP, } \\
\text { living in NHs }\end{array}$ & $\begin{array}{l}\text { Randomized study: } 284 \text { people were treated } \\
\text { for pain ( } 112 \text { received opioids; } 171 \\
\text { received non-opioids; } 57 \text { did not receive } \\
\text { any analgesics); investigators not blinded }\end{array}$ & $\begin{array}{l}\text { ES and SRM were correlated with changes in } \\
\text { EPCA-2 scores and GCS, opioid and non- } \\
\text { opioid dosages and demonstrated } \\
\text { satisfactory responsiveness of the EPCA-2 }\end{array}$ \\
\hline $\begin{array}{l}\text { Cohen- } \\
\text { Mansfield } \\
\text { 2008, [44] }\end{array}$ & $\begin{array}{l}\text { PAINE }^{\mathrm{a}} \\
\text { PADE }^{\mathrm{a}} \\
\text { PAINAD }^{\mathrm{b}} \\
\text { CNPI }^{\mathrm{b}} \\
\text { OPBAI }^{\mathrm{b}}\end{array}$ & $\begin{array}{l}36 \text { people with } \\
\text { dementia (MMSE } \\
\leq 20)\end{array}$ & $\begin{array}{l}\text { Before and after pain treatment evaluation } \\
\text { by self-report and proxy rating; pain } \\
\text { treatment with acetaminophen }(N=29) \text {, } \\
\text { combination acetaminophen }+ \text { oxycodone } \\
(N=6) \text { was compared to patients without } \\
\text { pain; investigators not blinded; small } \\
\text { sample size }\end{array}$ & $\begin{array}{l}\text { It is suggested that PAINE and PADE had } \\
\text { strongest detection in treatment effects }\end{array}$ \\
\hline $\begin{array}{l}\text { Rat et al. } \\
\text { 2011, [45] }\end{array}$ & Algoplus ${ }^{\circledR c}$ & $\begin{array}{l}109 \text { inpatients; } \\
\text { MMSE }<15 \text {, with } \\
\text { acute pain lasting } \\
<2 \text { weeks }\end{array}$ & $\begin{array}{l}\text { Multicenter, cross-sectional study including } \\
\text { five settings. Algoplus }{ }^{\circledR} \text { sensitivity to } \\
\text { change was tested by movement-induced } \\
\text { change during movement before and after } \\
\text { starting with WHO level I-III analgesics or } \\
\text { non-pharmacological treatment; } \\
\text { investigators blinded }\end{array}$ & $\begin{array}{l}109 \text { acute pain patients received level I drug } \\
(37 \%), \text { II drug }(30 \%) \text {, III drug }(23 \%), \\
\text { others }(3 \%) \text {. Paired comparisons by } \\
\text { Wilcoxon's signed-rank found Algoplus }{ }^{\circledR} \\
\text { scores before }(3.1 \pm 1.3) \text { and after } \\
\text { treatment }(1.6 \pm 1.1) \text { comparable with } \\
\text { changes on VAS and NRS }\end{array}$ \\
\hline $\begin{array}{l}\text { Husebo } \\
\text { et al. } \\
2014,[42]\end{array}$ & $\begin{array}{l}\text { MOBID-2 } \\
\text { Pain } \\
\text { Scale }\end{array}$ & $\begin{array}{l}352 \text { people with } \\
\text { advanced } \\
\text { dementia }\end{array}$ & $\begin{array}{l}\text { Multicenter, cluster randomized trial } \\
\text { including } 18 \mathrm{NHs;} \text { SPTP with paracetamol, } \\
\text { morphine, buprenorphine and/or } \\
\text { pregabalin; assessments at baseline, week } \\
2 \text { and 4; investigators blinded }\end{array}$ & $\begin{array}{l}\text { SEM and SDC in connection with MOBID-2 } \\
\text { Pain Scale measures indicate that the tool is } \\
\text { responsive to a decrease in pain after SPTP. } \\
\text { Satisfactory test-retest reliability } \\
\text { demonstrated. Change scores } \geq 3 \text { on total } \\
\text { and subscales are clinically relevant and } \\
\text { beyond measurement error }\end{array}$ \\
\hline
\end{tabular}

CNPI Checklist of Nonverbal Pain Indicators [94], EPCA-2 Elderly Pain Caring Assessment 2 [43], ES effect size, GCS global Clinical Score, MMSE Mini-Mental State Examination [95], MOBID Mobilization-Observation-Behavior-Intensity-Dementia [42], NH nursing home, NRS Numerical Rating Scale, $N V C$ - $O P$ non-verbally communicating older patients (age $\geq 65$ years), OPBAI Observational Pain Behavior Assessment Instrument [96], PADE Pain Assessment for the Dementing Elderly [97], PAINAD Pain Assessment in Advanced Dementia [91], PAINE Pain Assessment in Noncommunicative Elderly persons [98], SDC smallest detectable change, SEM standard error of measurement, SPTP stepwise protocol of treating pain, SRM standard response mean, VAS Visual Analog Scale, WHO World Health Organization

${ }^{\text {a }}$ Informant rating

b Observation rating

c Acute pain tool

signed-rank found Algoplus ${ }^{\circledR}$ scores of $3.1 \pm 1.3$ before and $1.6 \pm 1.1$ after treatment, comparable to changes on the Visual Analog Scale (VAS) and Numerical Rating Scale (NRS).

Our own group investigated the responsiveness of the Mobilization-Observation-Behavior-Intensity-Dementia (MOBID)-2 Pain Scale, using data from a cluster randomized clinical trial that included 352 Norwegian nursing home patients with advanced dementia and agitation [42]. Nursing home clusters were randomized to an SPTP with paracetamol, morphine, or buprenorphine, and/or pregabalin when neuropathic pain was suggested. This was a 12-week trial including a 4-week washout period after the SPTP. We followed the latest COSMIN recommendations to assess the responsiveness of the MOBID-2 Pain Scale. Test-retest reliability between baseline and week $2(N=163)$, and weeks 2 and $4(N=159)$ was examined in patients not expected to change (control), using the intraclass correlation coefficient $\left(\mathrm{ICC}_{2.1}\right)$, standard error of measurement (SEM), and smallest detectable change (SDC). Responsiveness was assessed by testing six a prioriformulated hypotheses about the relationship between change scores on the MOBID-2 Pain Scale and other outcomes. SEM and SDC indicated that the MOBID-2 Pain Scale is responsive to a decrease in pain after analgesic treatment. Satisfactory test-retest reliability was demonstrated. Change scores of $\geq 3$ on total and subscales were clinically relevant and beyond measurement error. 


\subsection{Which Analgesics are Efficacy-Tested by Clinical or Randomized Controlled Trials Including People with Dementia?}

\subsubsection{Paracetamol}

The use of paracetamol is recommended as the first-line therapy in people with dementia [14] and is to date the most used analgesic in nursing home patients worldwide [46, 47]. We found two studies conducted between 1998 and 2015 that investigated the efficacy of paracetamol (administered as monotherapy) on pain and/or behavioral disturbances in people with dementia (Table 2).

Chibnall et al. conducted a placebo-controlled, crossover trial for 4 weeks including 25 people with moderate to severe dementia [35]. Twenty-five patients received 4 weeks' paracetamol $1 \mathrm{~g}$ each $8 \mathrm{~h}$ and 4 weeks' placebo in random order, with a 1-week washout period; 23 patients completed both study phases. The effect was assessed with Dementia Care Mapping (DCM) and Cohen-Mansfield Agitation Inventory (CMAI) [35]. Results demonstrated that patients in the intervention phase were more active and interacted with each other, and spent more time in social surroundings. Paracetamol, however, did not ameliorate agitation, improve emotional well-being, or reduce the use of as-needed psychotropic medication. A validated pain assessment tool was not used.

Buffum et al. included 39 nursing home patients with advanced dementia and degenerative joint diseases in a placebo-controlled, crossover trial for 4 weeks (each arm lasted for 2 weeks) to investigate the impact of regularly scheduled analgesic treatment compared with as-needed administration [34]. Patients randomized to the intervention group received $2.6 \mathrm{~g}$ paracetamol in $24 \mathrm{~h}$. The treatment effect was assessed by the Discomfort ScaleDementia of Alzheimer Type (DS-DAT). No differences in pain and/or discomfort were found between the groups. It was suggested, however, that the paracetamol dosage was rather too low to ameliorate existing pain.

\subsubsection{Opioids}

We found two studies that investigated the efficacy of morphine and oxycodone (as a monotherapy) on neuropsychiatric symptoms, pain, and overall symptom management in people with dementia (Table 2). Manfredi et al. conducted a small double-blinded, crossover study with fixed treatment order for 8 weeks and included 47 people with moderate to severe dementia and behavioral disturbances from one nursing home [36]. Participants started with 4-week placebo treatment, and after a 1-day interval, a long-acting opioid (long-acting oxycodone $10 \mathrm{mg}$ every $12 \mathrm{~h}$, or for patients who could not swallow pills, long- acting morphine $20 \mathrm{mg}$ once a day, via a feeding tube) for the next 4 weeks. This approach did not include a randomized order of administration. The efficacy of opioid treatment on agitation was assessed by the CMAI, and no significant differences were found between the placebo and active phases in 25 participants who completed the study. Sub-analyses demonstrated, however, that 13 patients older than 84 years were less agitated. An observational pain assessment instrument was not used.

The second study, by Klapwijk et al. was an open prospective, observational follow-up trial in two Dutch nursing homes, and included 24 people with severe dementia who were expected to die within the next 7 days [39]. The judgment that the patient was perceived as dying was based on an estimation made by the treating physician and responsible nurse. Symptoms of discomfort, pain, and suffering were recorded twice a day using the Pain Assessment in Advanced Dementia (PAINAD), DS-DAT, and End-Of-Life in Dementia-Comfort assessment in Dying (EOLD-CAD) scales, and an adapted version of the Mini-Suffering State Examination (MSSE). Pain and distressing symptoms were treated with morphine. Each patient was observed several times (mean 4.3) until they died, demonstrating low intensity scores of pain and other symptoms such as dyspnea and fear. However, the direct effect of morphine on pain or other distressing symptoms, and the exact dosages, were not available.

\subsubsection{Lidocaine (1\% Solution)}

Benedetti et al. included 28 non-consecutive, communicative, home-dwelling patients with AD and 16 healthy volunteers matched for sex and age (mean 74 years) [40]. Participants were invited to take blood samples in the laboratory. This procedure is often described as a burning sensation and, thereby, patients are treated with the local anesthetic lidocaine ( $1 \%$ solution). In the open (expected) condition, the drug was applied in full view of the patient, whereas in the hidden (unexpected) condition, the same dose of drug was administered with the patient completely unaware that a local anesthetic was being applied. To justify the lack of lidocaine application, the patients were told that lidocaine would be applied or not. Patients rated their pain sensation on an NRS. Results demonstrated that AD patients showed reduced placebo component of the analgesic treatment. Loss of the placebo-related mechanism reduced treatment efficacy, such that a dose increase was necessary to produce adequate analgesia.

\subsubsection{Vitamin D}

Björkman et al. investigated the efficacy of vitamin D (cholecalciferol) treatment on pain during a 6-month, double- 
blind, placebo-controlled clinical trial [33]. The study participants were assessed by the Resident Assessment Instrument (RAI) and DS-DAT (Table 2). Two hundred and two nursing home patients with cognitive impairment were randomized to three groups, which were treated with 0 , 400, or 1200 IU cholecalciferol. Despite growing evidence of the potential benefit of vitamin D supplementation on pain intensity, the etiology was still vague after 6 months of treatment.

\subsubsection{Multi-Analgesic Drug Use}

We identified two studies that investigated multi-analgesic stepwise drug use to treat pain and/or neuropsychiatric symptoms in people with dementia (Table 3). Results were published in six different articles [7, 37, 38, 48-50]. The first study, by Hendriks et al. included 330 dying people with dementia from 34 Dutch nursing homes (2007-2011) [38]. The End of Life in Dementia (EOLD) study is an open, longitudinal, observational trial with a 3.5-year follow-up, until death. The responsible physicians performed assessments at baseline, semiannually, and 2 weeks after death. The occurrence of pain, dyspnea, and agitation were scored dichotomously (present/not present). The quality of life was assessed by the Quality of Life in Late-stage Dementia (QUALID) scale. Independent of the stage of dementia, $52 \%$ of the patients were registered as in pain in the last week of life. Most of the patients (77\%) received opioids $(90 \mathrm{mg} / 24 \mathrm{~h} ; 88 \%$ by injection); opioid monotherapy was applied to 43 , and $57 \%$ of the dying received morphine combined with paracetamol. The second publication by Hendriks and colleagues investigated the associations between the symptoms of pain and agitation, as well as between the stage of dementia and symptoms [48]. They found that the prevalence of pain ranged from $47 \%$ to $68 \%$ across the semiannual assessments, and increased to $78 \%$ in the last week of life. Symptom treatment changed in particular at the end of life. Pain was treated mostly with paracetamol (34-52\%), and at the end of life with parenteral opioids (44\%). Pain and agitation were common and frequently persisted in residents with dementia during nursing home stay, but symptom management intensified only at the end of life. The authors concluded that symptom control may be suboptimal starting at admission, and this requires a stronger focus on symptom control at earlier stages than only the last days or weeks of life.

Our own study, described earlier, was a cluster randomized clinical trial over 12 weeks, including 352 people with advanced dementia and neuropsychiatric symptoms from 18 nursing homes in Norway [37]. Patients randomized to intervention received an SPTP with paracetamol, morphine, buprenorphine, and/or pregabalin on the basis of ongoing treatment and individual needs. The MOBID-2 Pain Scale, CMAI, Neuropsychiatric Inventory-Nursing Home version (NPI-NH), and the Activities of Daily Living (ADL) scales were regularly used for data collection at baseline, weeks $2,4,8$, and 12 (weeks $8-12$ washout). Analyses found agitation reduced in the SPTP group after 4 weeks (average reduction $17 \% ; p<0.001$ ). Verbally agitated behaviors, physically non-aggressive behaviors, and aggressive behaviors improved significantly $(p<0.001, p=0.008, p=0.037$, respectively) after 8 weeks [50]. Mood syndrome improved $(p<0.001)$, including depression $(p=0.025)$, apathy $(p=0.017)$, sleep $(p=0.050)$, and appetite $(p=0.005)$, but not irritability $(p=0.092)$ and anxiety $(p=0.125)$ [49]. Pain was reduced in the SPTP group compared with control $(p<0.001)$ at week 8 , and MOBID-2 scores and agitation worsened during the washout period $(p=0.022)$. Examining different analgesic treatments, patients receiving paracetamol improved significantly in their ADL function $(p=0.022)[7]$.

\subsection{Which Primary and Secondary Outcome Measures Were Used?}

Five studies used a pain assessment instrument (NRS-selfrating, DS-DAT, MOBID-2 Pain Scale, RAI, and PAINAD) to investigate the efficacy of treating pain on pain intensity. Of these instruments, the MOBID-2 Pain Scale is the only instrument that has been tested for responsiveness in dementia patients [42] (Tables 2, 3). Some papers investigated the efficacy of treating pain on indirect measures, such as agitation, neuropsychiatric symptoms, quality of life, activity, and more general symptom control at the end of life (CMAI, NPI-NH, ADL, QUALID, DCM, EOLD-CAD). For the QUALID, a measure for quality of life in dementia, there has been only one study on responsiveness, which showed that it was not responsive to changed neuropsychiatric symptoms [51]. Quality of life was related to neuropsychiatric symptoms at baseline and after intervention, but not related to change scores. The instruments that measure neuropsychiatric symptoms (CMAI and NPI-NH) have been proven responsive to several (non-)pharmacological behavioral interventions. CMAI was also responsive to pain medication in one study [37]. In the same study sample, the NPI-NH subscale mood was also responsive to pain medication [7], as was the ADL measure [52]. We could not find other studies in which responsiveness was tested on the effect of pain medication in a dementia population.

The EOLD-CAD is an instrument that measures quality of dying and performs well in terms of validity, reliability, and feasibility [53]. However, there are no data on its responsiveness, or studies showing relevant differences 
after pain interventions. The DCM tool (a direct observation tool, developed for use in nursing homes) has been shown to be able to pick up changes in activity after an intervention with paracetamol, although no formal responsiveness test results are presented [35].

\section{Discussion}

This article adds to previous literature reviews of the assessment and treatment of pain in people with dementia [1, 12, 21, 54, 55], with a particular focus on studies investigating the direct efficacy of analgesics on pain intensity. In this context, it was also important to give an overview of responsive tools that are able to assess change in the total pain intensity score after initiation of the treatment. We further extended our search to studies that included dying people with dementia because it is widely acknowledged that dementia is a life-limiting disease with considerable pain and symptom burden in the last days and hours of life [31, 56, 57].

\subsection{Methodological Perspectives}

We found few studies employed a blinded, controlled trial design; they generally included few participants; and unfortunately, most of the publications did not sufficiently describe management of dropouts. The possible lack of statistical power and selection bias means that the studies in this review may have methodological and statistical biases. There was also a wide variation both in the interventions used and in the study designs.

Hence, a limitation with our systematic literature review is the limited possibility to compare the studies in terms of quality and methods. Conclusive recommendations based on aggregated evidence are at this point difficult to provide. In addition, with our strict inclusion criteria, we cannot exclude the possibility of publication bias.

\subsection{Responsiveness of Pain Assessment Tools}

Thus far, few studies have investigated the responsiveness of pain assessment tools used in people with dementia or non-communicating elderly people. Responsiveness is of critical importance for clinicians and researchers to be confident that an improvement in individual patients after treatment is not merely measurement error. The results of the few responsiveness studies of the single measurement tools are challenging to interpret because treatment groups were partly small with different treatment and measurement approaches.

The initial step to improve pain treatment is proper assessment by monitoring the change of pain intensity to the pain treatment intervention. We found four studies that reported the responsiveness of the observational tools EPCA-2 [43], MOBID-2 Pain Scale [42], PAINE and PADE [44], developed and tested for nursing home patients with dementia, and the Algoplus ${ }^{\circledR}$ for the evaluation of acute pain treatment in hospital [45]. However, the evidence to support the use of these pain tools is rather limited. Recent requirements by the COSMIN group recommended a checklist for assessing the methodological quality of health status measurement instruments to improve the psychometric property testing [41]. Until now, only the MOBID-2 Pain Scale followed the latest COSMIN recommendations and examined measurement error by the SEM and the SDC, which are important parameters for judging change scores in large clinical trials investigating the efficacy of analgesics on pain intensity [7]. To the best of our knowledge, there are currently no other instruments that have been responsiveness tested for use in dying people with dementia [53]. Consequently, the direct efficacy of pain and symptom management cannot currently be monitored by a responsive tool at the end of life in these individuals. Studies on responsive non-verbal pain assessment tools in the end-of-life setting are needed.

\subsection{Pain Assessment in Dementia}

Today, there is extensive ongoing work to develop and test appropriate pain assessment instruments for people with advanced dementia [58]. Most of the instruments are based on the assumption and recommendations of the American Geriatrics Society (AGS) Panel that pain can be expressed by changes in facial expression (e.g., frowning), vocalization and verbalization (e.g., groaning, mumbling), and body movements $[59,60]$. The AGS Panel suggests the following additional behavioral changes that may be triggered by untreated pain: changes in interpersonal interactions (e.g., aggressive, combative, resisting care), changes in activity patterns or routines (e.g., wandering, appetite, sleep), and mental status changes (e.g., crying, confusion, irritability). One important clinical and research dilemma is the difficulty of differentiation between typical behavior related to pain and behavior that is caused by the dementia process itself. These behaviors may present in a similar way for the observer [21]. Importantly, most scales are validated for ascertaining the presence of pain, but not the pain intensity. In most scales, we do not know if scoring a higher number of behavioral items also means more pain. In addition, in these scales, all behavioral items that can be scored are given the same weight. No studies have tried to differentiate pain intensity between these items, and therefore, we cannot say if, for instance, guarding, frowning, or screaming is an indication of more pain. Consequently, we need more research on the responsiveness of 
these behavioral pain observation scales, and these should not only be studied on the change of sum scores. Sensitive responsive instruments are needed for the evaluation of pain treatment, and they will also require weighing of the different behavioral signs.

The psychometric property developments (validity, reliability, and responsiveness) of available instruments are ongoing tasks. General agreement exists that the assessment of pain in people with dementia should be based on the observation of the patient's pain behavior during activities of daily living and/or gentle guided standardized movements, because pain avoidance may mask pain behavior $[21,58,60-62]$. The interpretation of pain behavior into pain intensity scores is a necessary task because the presence of the behavior does not automatically mean clinically significant pain intensity. As demonstrated by the MOBID-2 Pain Scale, the differentiation between pain related to the muscle-skeletal system and internal organs, head, and skin is relevant for the clinician, since different locations of pain may require different treatment approaches. The nursing staff need to have their daily observations systematized and corroborated by a validated total pain intensity score. A cut-point will indicate when to contact the physician who continues further evaluations and treatment.

\subsection{Pharmacological Management of Pain in Dementia}

We identified eight randomized prospective treatment trials with a comparator group or open studies with more than ten participants that investigated the direct effect of analgesics on pain and/or neuropsychiatric symptoms including people with dementia. Importantly, only four of these studies used a pain assessment instrument to monitor the analgesic treatment effect on pain intensity. There is limited evidence as to the effect of using paracetamol and morphine. We are not aware of studies that included treatment with NSAIDs, tramadol, or codeine. However, Stein et al. conducted a 3-month, randomized, controlled trial to investigate the efficacy of an educational program for nursing home staff to improve pain treatment and indirectly reduce the use of NSAIDs [63]. The education of staff resulted in a reduction of the time of NSAID use from 7 to 2 days and from 7 to 6 days in the control group. Interestingly, the reduction did not worsen the pain [63]. Buprenorphine and pregabalin have only been used in one large, cluster randomized, controlled trial that offered a stepwise approach to treating pain [37].

Although paracetamol is traditionally the most used analgesic drug in nursing homes [46], we found only two trials that investigated the efficacy of this treatment on pain intensity and/or behavioral disturbances in people with dementia. Both studies were randomized, placebo-controlled, crossover trials and demonstrated inconsistent results. Chibnall included 25 patients who received 4 weeks of paracetamol ( $3 \mathrm{~g} /$ day) and 4 weeks of placebo [35]. Patients randomized to the intervention group were more active and spent more time in social surroundings. The efficacy on pain intensity scores was not investigated by a validated pain tool. Buffum et al. investigated the efficacy of regularly scheduled paracetamol for discomfort in people with advanced dementia over 4 weeks (2-week treatment with scheduled vs. 2-week treatment with asneeded administration) [34]. Assessed by the DS-DAT, regular use of paracetamol did not confer any benefit compared with as-needed administration of the drug. Interestingly, the authors found that a dosage of $2600 \mathrm{mg}$ paracetamol per day was not adequate to ameliorate pain in connection with degenerative joint disease. Agitation, as well, was not reduced by the treatment [34]. The only trial that directly investigated the effect of morphine monotherapy on pain intensity was a placebo-controlled, crossover trial including 47 nursing home patients with dementia [36]. Manfredi et al. found reduction of agitation in patients aged 85 or older, suggesting that opioids used in very old patients may be a beneficial treatment for agitation [36]. The impact on pain intensity scores was not assessed. All the three studies were small and underpowered crossover trials; the results are challenging to interpret because of this and because of the lack of pain assessment instruments or low doses of paracetamol.

Two other placebo-controlled trials need attention because of their robust methodologies, sample sizes, and innovative research questions. Benedetti et al. included 28 people with mild to moderate $\mathrm{AD}$; they were younger (mean 74 years) and lived at home [40]. Using lidocaine gel during a painful procedure, the investigators demonstrated a reduction of the placebo component in patients with dementia. It was concluded that pain treatment should be adjusted to compensate for the loss of placebo effect [40]. The other study, by Björkman et al. included 202 people with dementia and explored the efficacy of treating pain with vitamin D supplementation in three groups [33]. Pain was assessed by three different pain tools (RAI, PAINAD, and DS-DAT). However, the authors did not find any effect of vitamin D on pain or pain behavior; neither prevalence of painlessness nor pain scores changed after vitamin D treatment [33]. One large cluster randomized study supported guidelines from the AGS panel by a stepwise protocol of treating pain in people with dementia demonstrating clinically relevant agitation and pain [37]. The study demonstrated that low doses of morphine and buprenorphine were well tolerated and ameliorated pain in people with dementia. Also paracetamol was a safer alternative medication than psychotropic drugs and 
improved activities of daily living [7]. In people with neuropathic pain, pregabalin significantly reduced the pain intensity scores [7]. Finally, the mood syndrome was improved by individual pain treatment [49]. However, this study was not placebo controlled and the results should be validated by a well powered trial using active and placebo analgesics.

\subsection{Pain Treatment in Dying Patients with Dementia}

The needs of people with dementia at the end of life may be different from those with different diseases $[64,65]$. Thus, it was important for us to also include two observational studies of palliative treatment that described the effectiveness of analgesics on pain intensity scores or symptom burden in these individuals. The prospective observational study by Klapwijk et al. observed 24 patients from the day when the person was perceived as dying and to the day of death [39]. All patients received morphine subcutaneously. Although the most used pain assessment instruments (PAINAD, DS-DAT) were not validated for this end-of-life situation, the multidisciplinary team was pleased with the pain and symptom relieving effect of the treatment [39]. The study by Hendriks et al. included 330 dying patients, who were retrospectively (up to 2 weeks after death) assessed by 103 elderly care physicians [38, 48]. Fifty-two percent of the patients were affected by pain, which was dichotomized as present or not, and were treated with $90 \mathrm{mg}$ morphine $/ 24 \mathrm{~h}$ on the day of death. Both studies are groundbreaking contributions to the assessment and treatment of pain and burdensome symptoms in dying people with dementia, although the study by Klapwijk et al. was somewhat underpowered and the study by Hendriks et al. retrospective. Both studies were observational; thus, the direct effect of the analgesics was difficult to interpret.

Pain research in dying people with dementia is of particular interest for several reasons. First, research including elderly patients often excludes people with dementia and, until now, especially dying people with dementia [66-68]. Further, palliative care for cancer patients cannot necessarily be transferred to dying people with dementia [69]. These individuals are neither able to describe the pain nor the pain treatment effect or side effects. Third, the nature of dementia leads to reduction of the neurotransmitter acetylcholine, responsible for neuromuscular junction and muscle activities, and the autonomic, sympathetic, and parasympathetic nervous system. Consequently, drugs that affect the cholinergic systems (anticholinergic drugs) can have very dangerous effects, ranging from paralysis to convulsions [70]. Importantly, most of the drugs used in palliative care (morphine, scopolamine, or midazolam) to relieve pain and distressing symptoms have anticholinergic side effects. A self-fulfilling prophecy may be the case when a patient with dementia is wrongly identified as dying and then receives this treatment, hastening the death. These challenges argue for the urgent need for clinical studies, disease-specific guidelines, and implementation of research-based knowledge [31].

\subsection{Analgesic Drug Prescription}

Fifteen years ago, researchers echoed that people with advanced dementia have a substantial risk for undetected or undertreated pain. Morrison and Siu examined the treatment of pain following hip fractures in a prospective cohort study and found that people with advanced dementia received only one-third the amount of morphine compared with cognitively intact patients [71]. Another study, by Closs et al. explored analgesic prescription according to cognitive status in nursing home patients and found no differences in pain scores, but administration of opioid and non-opioid analgesics were highest for residents without dementia [72]. Results are supported by a more recent study from the USA, which identified nursing home patients with similar pain-related diagnoses; patients without dementia received significantly more opioids compared with those with cognitive impairment, despite higher pain intensity scores based on interviews and use of a numeric rating scale [73]. In addition, there are many other studies that confirm the under-treatment of pain in people with dementia in the community, residential care settings, nursing homes, and hospitals [3].

Nevertheless, times are changing. Recent prevalence studies demonstrated new trends of analgesic drug use. In the Kungsholmen Study, $46 \%$ of 2610 people with dementia used at least one analgesic drug compared with $25 \%$ of those without dementia [5]. Another nationwide Swedish study demonstrated that centenarians ( $>100$ years) used more analgesics, anxiolytics, and hypnotics compared with younger patients [74]. Indeed, the use of analgesics in 546 older people ( $>85$ years), with and without dementia, at home and in the nursing home, was high in Sweden and Finland. People with dementia used more paracetamol and likewise amounts of opioids compared with those without cognitive impairment [75]. In addition, the use of strong opioids (e.g., transdermal fentanyl) was higher in people with dementia in Finland [76]. In Danish nursing home patients, $41 \%$ of all elderly receive opioids, and those with dementia and nursing home residents have a higher chance of receiving opioids [77].

Most importantly, none of these studies reported better accuracy of pain assessment procedures, higher or improved pain management effects, or side effects of the treatment. This is of key importance because, based on Rhode Island Medicaid pharmacy claims about nursing 
home data, the US Food and Drug Administration (FDA) warned against morbidity and death related to the launch of long-acting opioids and the fentanyl transdermal system in opioid-naive people with AD [78]. The authors found these drugs more frequently prescribed in those with advanced age and increased cognitive impairment. Recently, our own research demonstrated almost a doubling of analgesics, from 35 to $58 \%$, in Norwegian nursing homes during the last 10 years (2000-2011) [47]. Results support recent trend analyses, which found that the use of analgesic drugs has been increasing rapidly over the last 8 years [79]. It seems a paradox, however, that the crucial question remains: does the right patient receive the right medication for the proper indication at the right time [1]? Although few of the recent studies suggest that analgesics are systematically under-prescribed, there is no information whatsoever on assessment, indication, evaluation, benefits, and harms [80]. Thus, attaining a competent balance between under- and overuse of analgesics in people with dementia remains the most important challenge in this field.

Getting older is associated with a progressive decline of organ function, including typical changes such as reduced body mass index and lower body water percentage compared with a higher fat mass percentage [70]. This is often combined with latent insufficiency of liver, a lower glomerular filtration rate of the kidneys, and cognitive function and biological alterations (such as a lower total brain mass) that impact the pharmacodynamics and pharmacokinetics of the drug. The absorption, distribution, and elimination of the drugs are altered, especially when several drugs are combined as a result of polypharmacy. Beers Criteria highlight a greater sensitivity to adverse events and higher risks of side effects in these people [81]. However, we did not find studies that investigated pharmacodynamics and pharmacokinetics of analgesic drugs in people with dementia. Being aware of the potential lack of placebo effect in dementia and the potential effects of dementia on the brain-blood barrier certainly warrants those studies.

\subsection{Pain Treatment Recommendations}

Although best practice recommendations for the assessment and treatment of pain in elderly people are available $[12,14,23,59,60,82-84]$, clinicians in the nursing home setting may not routinely follow-or even be aware of these guidelines. These expert groups predominantly endorse some specific drugs: paracetamol, strong opioids, and anticonvulsants [12, 14, 23, 59, 60, 82-84]. Results of this review, however, underline the weak evidence base for all analgesics in dementia. Although the process of developing new evidence, the foundation of clinical guidelines and implementation, is not always linear, there is still a lack of well powered randomized controlled trials, and this prevents definite clinical guidelines being implemented among clinical staff [85]. There is some evidence that paracetamol can be recommended, as it is relatively safe and some studies underline its efficacy. We therefore stress the need for placebo-controlled, randomized studies not only investigating the efficacy of NSAIDs, anticonvulsants, and morphine, but also withdrawal studies including patients who no longer are in need of these drugs.

\section{Conclusion}

To conclude, this review underlines the serious situation that pain management in especially advanced dementia is in at this moment: thorough pain assessment is poorly implemented and observational pain instruments have been scarcely tested for responsiveness; therefore, effective evaluation of pain management strategies is lacking and evidence for efficacy and safety of analgesics is largely missing. We emphasize the critical need for future research with well powered pain medication trials in persons with moderate to severe dementia using pain assessment tools that have been tested for responsiveness. Furthermore, there is a high need for studies investigating the end-of-life care in this population.

Acknowledgments The authors would like to thank medical librarian Regina Küfner Lein at the University of Bergen for assistance and advice in performing the systematic search.

\section{Compliance with Ethical Standards}

Conflict of interest Bettina Husebo, Wilco Achterberg, and Elisabeth Flo have no conflict of interest to declare.

Funding The Research Council of Norway (Sponsor's Protocol Code $222113)$ is funding the postdoctoral grant $(50 \%)$ for Elisabeth Flo. Bettina Husebo would like to thank the Norwegian Government and the GC Rieber Foundation for supporting her time for this work. The open access payment was funded by the University of Bergen, Norway.

Open Access This article is distributed under the terms of the Creative Commons Attribution-NonCommercial 4.0 International License (http://creativecommons.org/licenses/by-nc/4.0/), which permits any noncommercial use, distribution, and reproduction in any medium, provided you give appropriate credit to the original author(s) and the source, provide a link to the Creative Commons license, and indicate if changes were made.

\section{References}

1. Corbett A, Husebo BS, Malcangio M, et al. Assessment and treatment of pain in people with dementia. Nat Rev Neurol. 2012;8:264-74. 
2. Shega JW, Hougham GW, Stocking CB, Cox-Hayley D, Sachs GA. Pain in community-dwelling persons with dementia: frequency, intensity, and congruence between patient and caregiver report. J Pain Symptom Manage. 2004;28:585-92.

3. Achterberg WP, Gambassi G, Finne-Soveri H, et al. Pain in European long-term care facilities: cross-national study in Finland, Italy and The Netherlands. Pain. 2010;148:70-4.

4. Husebo BS, Strand LI, Moe-Nilssen R, et al. Who suffers most? Dementia and pain in nursing home patients: a cross-sectional study. JAMDA. 2008;9:427-33.

5. Haasum Y, Fastbom J, Fratiglioni L, Kareholt I, Johnell K. Pain treatment in elderly persons with and without dementia: a population-based study of institutionalized and home-dwelling elderly. Drugs Aging. 2011;28:283-93.

6. Lövheim H, Karlsson S, Gustafson Y. The use of central nervous system drugs and analgesics among very old people with and without dementia. Pharmacoepidemiol Drug Saf. 2008;17:912-8.

7. Sandvik RK, Selbaek G, Seifert R, et al. Impact of a stepwise protocol for treating pain on pain intensity in nursing home patients with dementia: a cluster randomized trial. Eur J Pain. 2014;18(10):1490-500.

8. Tune LE. Anticholinergic effects of medication in elderly patients. J Clin Psychiatry. 2001;62(Suppl 21):11-4.

9. Melis RJF, Marengoni A, Rizzuto D, et al. The influence of multimorbidity on clinical progression of dementia in a population-based cohort. PLoS ONE. 2013;8:e84014.

10. Monroe TB, Parish A, Mion LC. Decision factors nurses use to assess pain in nursing home residents with dementia. Arch Psychiatr Nurs. 2015;29:316-20.

11. Husebo BS, Flo E, Aarsland D, et al. COSMOS-improving the quality of life in nursing home patients: protocol for an effectiveness-implementation cluster randomized clinical hybrid trial. Implement Sci. 2015;10:131.

12. Achterberg WP, Pieper MJ, van Dalen-Kok AH, et al. Pain management in patients with dementia. Clin Interv Aging. 2013;8:1471-82.

13. Flo E, Gulla C, Husebo BS. Effective pain management in patients with dementia: benefits beyond pain? Drugs Aging. 2014;31:863-71.

14. Herr K, Coyne PJ, McCaffery M, Manworren R, Merkel S. Pain assessment in the patient unable to self-report: position statement with clinical practice recommendations. Pain Manag Nurs. 2011;12:230-50.

15. Dartigues JF. Alzheimer's disease: a global challenge for the $21 \mathrm{st}$ century. Lancet Neurol. 2009;8:1082-3.

16. Scherder EJ, Plooij B. Assessment and management of pain, with particular emphasis on central neuropathic pain, in moderate to severe dementia. Drugs Aging. 2012;29:701-6.

17. Scherder E, Oosterman J, Swaab D, et al. Recent developments in pain in dementia. BMJ. 2005;330:461-4.

18. Lyketsos CG, Lopez O, Jones B, et al. Prevalence of neuropsychiatric symptoms in dementia and mild cognitive impairment: results from the cardiovascular health study. JAMA. 2002;288:1475-83

19. Cummings JL, Mega M, Gray K, et al. The Neuropsychiatric Inventory: comprehensive assessment of psychopathology in dementia. Neurology. 1994;44:2308-14.

20. Husebo BS, Ballard C, Aarsland D. Pain treatment of agitation in patients with dementia: a systematic review. Int J Geriatr Psychiat. 2011;26:1012-8.

21. Husebo BS, Corbett A. Dementia: pain management in dementiathe value of proxy measures. Nat Rev Neurol. 2014;10(6):313-4.

22. Stolee P, Hillier LM, Esbaugh J, et al. Instruments for the assessment of pain in older persons with cognitive impairment. JAGS. 2005;53:319-26.
23. Herr K, Bjoro K, Decker S. Tools for assessment of pain in nonverbal older adults with dementia: a state-of-the-science review. J Pain Sympt Manage. 2006;31:170-92.

24. Zwakhalen S, Hamers J, Abu-Saad H, Berger M. Pain in elderly people with severe dementia: a systematic review of behavioural pain assessment tools. BMC Geriatr. 2006;6:3.

25. Hadjistavropoulos TP, Herr KP, Turk DCP, et al. An interdisciplinary expert consensus statement on assessment of pain in older persons. Clin J Pain. 2007;23:S1-43.

26. Chapman CR. Progress in pain assessment: the cognitively compromised patient. Curr Opin Anaesthesiol. 2008;21:610-5.

27. Juyoung P, Castellanos-Brown K, Belcher J. A review of observational pain scales in nonverbal elderly with cognitive impairments. Res Soc Work Pract. 2010;20:651-64.

28. Van Herk R, Van Dijk M, Baar FP, Tibboel D, De Wit R. Observation scales for pain assessment in older adults with cognitive impairments or communication difficulties. Nurs Res. 2007;56:34-43.

29. Herr K. Pain assessment strategies in older patients. J Pain. 2011;12:S3-13.

30. Vandervoort A, Van den Block L, van der Steen JT, et al. Nursing home residents dying with dementia in Flanders, Belgium: a nationwide postmortem study on clinical characteristics and quality of dying. J Am Med Dir Assoc. 2013;14:485-92.

31. van der Steen JT, Radbruch L, Hertogh CM, et al. White paper defining optimal palliative care in older people with dementia: a Delphi study and recommendations from the European Association for Palliative Care. Palliat Med. 2014;28:197-209.

32. Corbett A, Husebo BS, Achterberg WP, et al. The importance of pain management in older people with dementia. Br Med Bull. 2014;111:139-48.

33. Björkman M, Sorva A, Tilvis R. Vitamin D supplementation has no major effect on pain or pain behavior in bedridden geriatric patients with advanced dementia. Aging Clin Exp Res. 2008;20:316-21.

34. Buffum MD, Sands L, Miaskowski C, Brod M, Washburn A. A clinical trial of the effectiveness of regularly scheduled versus asneeded administration of acetaminophen in the management of discomfort in older adults with dementia. J Am Geriatr Soc. 2004;52:1093-7.

35. Chibnall JT, Tait RC, Harman B, Luebbert RA. Effect of acetaminophen on behavior, well-being, and psychotropic medication use in nursing home residents with moderate-to-severe dementia. J Am Geriatr Soc. 2005;53:1921-9.

36. Manfredi PL, Breuer B, Wallenstein S, et al. Opioid treatment for agitation in patients with advanced dementia. Int $\mathbf{J}$ Geriatr Psychiat. 2003;18:700-5.

37. Husebo BS, Ballard C, Sandvik R, Nilsen OB, Aarsland D. Efficacy of treating pain to reduce behavioural disturbances in residents of nursing homes with dementia: cluster randomised clinical trial. BMJ. 2011;343:d4065.

38. Hendriks SA, Smalbrugge M, Hertogh CM, van der Steen JT. Dying with dementia: symptoms, treatment, and quality of life in the last week of life. J Pain Sympt Manage. 2014;47:710-20.

39. Klapwijk MS, Caljouw MA, van Soest-Poortvliet MC, van der Steen JT, Achterberg WP. Symptoms and treatment when death is expected in dementia patients in long-term care facilities. BMC Geriatr. 2014;14:99.

40. Benedetti F, Arduino C, Costa S, et al. Loss of expectation-related mechanisms in Alzheimer's disease makes analgesic therapies less effective. Pain. 2006;121:133-44.

41. Mokkink LB, Terwee CB, Patrick DL, et al. The COSMIN checklist for assessing the methodological quality of studies on measurement properties of health status measurement instruments: an international Delphi study. QoL Res. 2010;19:539-49. 
42. Husebo BS, Ostelo R, Strand LI. The MOBID-2 pain scale: reliability and responsiveness to pain in patients with dementia. Eur J Pain. 2014;18(10):1419-30.

43. Morello R, Jean A, Alix M, Sellin-Peres D, Fermanian J. A scale to measure pain in non-verbally communicating older patients: the EPCA-2 Study of its psychometric properties. Pain. 2007;133:87-98.

44. Cohen-Mansfield J. The relationship between different pain assessments in dementia. Alzheimer Dis Assoc Disord. 2008;22:86-93.

45. Rat P, Jouve E, Pickering G, et al. Validation of an acute painbehavior scale for older persons with inability to communicate verbally: Algoplus. Eur J Pain. 2011;15(198):e1-10.

46. Tan EC, Jokanovic N, Koponen MP, et al. Prevalence of analgesic use and pain in people with and without dementia or cognitive impairment in aged care facilities: a systematic review and meta-analysis. Curr Clin Pharmacol. 2015;10:194-203.

47. Sandvik RK, Selbaek G, Kirkevold O, Aarsland D, Husebo BS. Analgesic prescribing patterns in Norwegian nursing homes from 2000 to 2011: trend analyses of four data samples. Age Aging. 2016;45(1):54-60.

48. Hendriks SA, Smalbrugge M, Galindo-Garre F, Hertogh CMPM, van der Steen JT. From admission to death: prevalence and course of pain, agitation, and shortness of breath, and treatment of these symptoms in nursing home residents with dementia. J Am Med Dir Assoc. 2015;16:475-81.

49. Husebo BS, Ballard C, Fritze F, Sandvik RK, Aarsland D. Efficacy of pain treatment on mood syndrome in patients with dementia: a randomized clinical trial. Int $\mathbf{J}$ Geriatr Psychiat. 2014;29:828-36.

50. Husebo BS, Ballard C, Cohen-Mansfield J, Seifert R, Aarsland D. The response of agitated behavior to pain management in persons with dementia. Am J Geriatr Psychiatry. 2014;22:708-17.

51. Benhabib H, Lanctot KL, Eryavec GM, Li A, Herrmann N. Responsiveness of the QUALID to improved neuropsychiatric symptoms in patients with Alzheimer's disease. Can Geriatr J. 2013;16:180-5.

52. Mahoney FI, Barthel DW. Functional evaluation: the Barthel index. Md State Med J. 1965;14:61-5.

53. van Soest-Poortvliet MC, van der Steen JT, Zimmerman S, et al. Selecting the best instruments to measure quality of end-of-life care and quality of dying in long term care. J Am Med Dir Assoc. 2013;14:179-86.

54. Pieper MJC, van Dalen-Kok AH, Francke AL, et al. Interventions targeting pain or behaviour in dementia: a systematic review. Age Res Rev. 2013;12:1042-55.

55. McClam TD, Marano CM, Rosenberg PB, Lyketsos CG. Interventions for neuropsychiatric symptoms in neurocognitive impairment due to Alzheimer's disease: a review of the literature. Harvard Rev Psychiatry. 2015;23:377-93.

56. Mitchell SL. Advanced dementia. N Engl J Med. 2015;372:2533-40.

57. Halpern SD. Toward evidence-based end-of-life care. N Engl J Med. 2015;373:2001-3.

58. Corbett A, Achterberg W, Husebo B, et al. An international road map to improve pain assessment in people with impaired cognition: the development of the Pain Assessment in Impaired Cognition (PAIC) meta-tool. BMC Neurol. 2014;14:229.

59. AGS. The management of persistent pain in older persons. J Am Geriatr Soc. 2002;50:S205-24.

60. AGS. Pharmacological management of persistent pain in older persons. Pain Med. 2009;10:1062-83.

61. Horgas AL, Elliott AF, Marsiske M. Pain assessment in persons with dementia: relationship between self-report and behavioral observation. J Am Geriatr Soc. 2009;57:126-32.
62. Lethem J, Slade PD, Troup JDG, Bentley G. Outline of a fearavoidance model of exaggerated pain perception-I. BRAT. 1983;21:401-8.

63. Stein CM, Griffin MR, Taylor JA, et al. Educational program for nursing home physicians and staff to reduce use of non-steroidal anti-inflammatory drugs among nursing home residents: a randomized controlled trial. Med Care. 2001;39:436-45.

64. Raymond M, Warner A, Davies N, et al. Palliative and end of life care for people with dementia: lessons for clinical commissioners. Prim Health Care Res Dev. 2014;15:406-17.

65. Kendall M, Carduff E, Lloyd A, et al. Different experiences and goals in different advanced diseases: comparing serial interviews with patients with cancer, organ failure, or frailty and their family and professional carers. J Pain Sympt Manage. 2015;50:216-24.

66. Bayer A, Tadd W. Unjustified exclusion of elderly people from studies submitted to research ethics committee for approval: descriptive study. BMJ. 2000;321:992-3.

67. Taylor JS, DeMers SM, Vig EK, Borson S. The disappearing subject: exclusion of people with cognitive impairment and dementia from geriatrics research. J Am Geriatr Soc. 2012;60:413-9.

68. Monroe TB, Herr KA, Mion LC, Cowan RL. Ethical and legal issues in pain research in cognitively impaired older adults. Int $\mathrm{J}$ Nurs Stud. 2013;50:1283-7.

69. Kendall M, Buckingham S, Ferguson S, Hewitt N, Pinnock H. We need to stop looking for something that is not there. NPJ Prim Care Respir Med. 2014;24:14031.

70. McLachlan AJ, Bath S, Naganathan V, et al. Clinical pharmacology of analgesic medicines in older people: impact of frailty and cognitive impairment. Br J Clin Pharmacol. 2011;71:351-64.

71. Morrison RS, Siu AL. A comparison of pain and its treatment in advanced dementia and cognitively intact patients with hip fracture. J Pain Sympt Manage. 2000;19:240-8.

72. Closs SJ, Barr B, Briggs M. Cognitive status and analgesic provision in nursing home residents. Br J Gen Pract. 2004;54:919-21.

73. Monroe TB, Misra SK, Habermann RC, et al. Pain reports and pain medication treatment in nursing home residents with and without dementia. Geriatr Gerontol Int. 2014;14:541-8.

74. Wastesson JW, Parker MG, Fastbom J, Thorslund M, Johnell K. Drug use in centenarians compared with nonagenarians and octogenarians in Sweden: a nationwide register-based study. Age Ageing. 2012;41:218-24.

75. Lovheim H, Karlsson S, Gustafson Y. The use of central nervous system drugs and analgesics among very old people with and without dementia. Pharmacoepidemiol Drug Saf. 2008;17:912-8.

76. Hamunen K, Paakkari P, Kalso E. Trends in opioid consumption in the Nordic countries 2002-2006. Eur J Pain. 2009;13:954-62.

77. Jensen-Dahm C, Gasse C, Astrup A, Mortensen PB, Waldemar G. Frequent use of opioids in patients with dementia and nursing home residents: a study of the entire elderly population of Denmark. Alz Dement. 2015;11:691-9.

78. Dosa DM, Dore DD, Mor V, Teno JM. Frequency of long-acting opioid analgesic initiation in opioid-naive nursing home residents. J Pain Sympt Manage. 2009;38:515-21.

79. Pitkala KH, Juola AL, Hosia H, et al. Eight-year trends in the use of opioids, other analgesics, and psychotropic medications among institutionalized older people in Finland. J Am Med Dir Assoc. 2015;16:973-8.

80. Achterberg W. Pain management in long term care: are we finally on the right track? Age Ageing. 2016;45(1):7-8.

81. American Geriatrics Society updated Beers Criteria for potentially inappropriate medication use in older adults. J Am Geriatr Soc. 2012;60:616-31.

82. American Geriatrics Society (AGS). The management of chronic pain in older persons: AGS panel on chronic pain in older persons. AGS. J Am Geriatr Soc. 1998;46:635-51. 
83. Herr K. Neuropathic pain: a guide to comprehensive assessment. Pain Manag Nurs. 2004;5:9-18.

84. Abdulla A, Bone M, Adams N, et al. Evidence-based clinical practice guidelines on management of pain in older people. Age Ageing. 2013;42:151-3.

85. Craig P, Dieppe P, Macintyre S, et al. Developing and evaluating complex interventions: the new Medical Research Council guidance. Int J Nurs Stud. 2013;50:587-92.

86. Cohen-Mansfield J. Agitated behaviors in the elderly. II. Preliminary results in the cognitively deteriorated. J Am Geriatr Soc. 1986;34:722-7.

87. Hurley AC, Volicer BJ, Hanrahan PA, Houde S, Volicer L. Assessment of discomfort in advanced Alzheimer patients. Res Nurs Health. 1992;15:369-77.

88. Volicer L, Hurley AC, Blasi ZV. Scales for evaluation of end-oflife care in dementia. Alzheimer Dis Assoc Disord. 2001;15:194-200.

89. Dubois B, Slachevsky A, Litvan I, Pillon B. The FAB: a frontal assessment battery at bedside. Neurology. 2000;55:1621-6.

90. Reisberg B, Ferris SH, de Leon MJ, Crook T. Global Deterioration Scale (GDS). Psychopharmacol Bull. 1988;24:661-3.
91. Warden V, Hurley AC, Volicer L. Development and psychometric evaluation of the Pain Assessment in Advanced Dementia (PAINAD) scale. J Am Med Dir Assoc. 2003;4:9-15.

92. Wood S, Cummings JL, Ming-Ann H, Barclay T, et al. The use of the neuropsychiatric inventory in nursing home residents: characterization and measurement. Am J Geriatr Psychiatry. 2000;8:75-83.

93. Weiner MF, Martin-Cook K, Svetlik DA, et al. The quality of life in late-stage dementia (QUALID) scale. J Am Med Dir Assoc. 2000;1:114-6.

94. Feldt KS. The Checklist of nonverbal pain indicators (CNPI). Pain Manag Nurs. 2000;1:13-21.

95. Folstein MF, Folstein SE, McHugh PR. "Mini-mental state". A practical method for grading the cognitive state of patients for the clinician. J Psychiatr Res. 1975;12:189-98.

96. Teske K, Daut RL, Cleeland CS. Relationships between nurses' observations and patients' self-reports of pain. Pain. 1983;16:289-96.

97. Villanueva MR, Smith TL, Erickson JS, Lee AC, Singer CM. Pain assessment for the dementing elderly (PADE): reliability and validity of a new measure. J Am Med Dir Assoc. 2003;4:1-8.

98. Cohen-Mansfield J. Pain assessment in noncommunicative elderly persons-PAINE. Clin J Pain. 2006;22:569-75. 